\title{
Development of a clinically relevant symptom index to assess patients with chronic orchialgia/chronic scrotal content pain
}

\author{
Alan Scott Polackwich ${ }^{1}$, Hans Chin Arora ${ }^{2}$, Jianbo Li ${ }^{2}$, Laurence Levine ${ }^{3}$, Bayo Tojuola ${ }^{4}$, Sijo Parekattil ${ }^{4}$, \\ Daniel A. Shoskes ${ }^{2}$ \\ ${ }^{1}$ Columbia University Department of Urology at Mount Sinai Medical Center, Miami Beach, FL, USA; ${ }^{2}$ Department of Urology, Cleveland Clinic, \\ Cleveland, OH, USA; ${ }^{3}$ Division of Urology, Department of General Surgery, Rush University Medical Center, Chicago, IL, USA; ${ }^{4}$ The Personalized \\ Urology \& Robotics Clinic, Clermont, FL, USA \\ Contributions: (I) Conception and design: S Parekattil, DA Shoskes; (II) Administrative support: S Parekattil, DA Shoskes; (III) Provision of study \\ material or patients: B Tojuola, S Parekattil, DA Shoskes; (IV) Collection and assembly of data: AS Polackwich, HC Arora, B Tojuola, S Parekattil, \\ DA Shoskes; (V) Data analysis and interpretation: J Li, L Levine, S Parekattil, DA Shoskes; (VI) Manuscript writing: All authors; (VII) Final approval \\ of manuscript: All authors. \\ Correspondence to: Daniel A. Shoskes, MD. Department of Urology, Glickman Urological and Kidney Institute, Cleveland Clinic, 9500 Euclid Avenue, \\ Q10-1, Cleveland, OH 44195, USA. Email: dshoskes@gmail.com.
}

Background: The purpose of this study was to develop a candidate symptom index for men with an established diagnosis of chronic orchialgia.

Methods: Based on interviews with patients and providers, we developed a 70 -item questionnaire that focused on seven areas of orchialgia symptoms: pain, location, urinary symptoms, sexual dysfunction, medical history and quality of life (QOL) impact. The questionnaire was completed by patients at two medical centers. Cluster analysis was performed with the software package R (3.2.1).

Results: A total of 113 men completed the survey. Median symptom duration was 12 months (range, 3-336 months). Outside the testicle, pain was felt in the spermatic cord (66\%), groin (66\%), penis (24\%), suprapubic region (38\%), flank (31\%), thigh (32\%), abdomen (36\%) and perineum $(35 \%)$. Bother scores were high only for testicle and spermatic cord pain. Urinary frequency was common (54\%) but not bothersome. Sexual dysfunction was common: $55 \%$ had erectile dysfunction, $56 \%$ had decreased libido and $39 \%$ had painful ejaculation with high bother for all. By cluster analysis, QOL parameters clustered tightly with minimal pain level, pain at night, burning pain, distribution to spermatic cord and groin, erectile dysfunction and premature ejaculation.

Conclusions: Men with chronic orchialgia have a high incidence of associated symptoms. Most bothersome symptoms with highest QOL impact include burning pain, pain at night, radiation to groin and spermatic cord, erectile dysfunction and low libido. Based on these findings, we have created a candidate orchialgia symptom index with domains of pain, sexual symptoms and QOL that will undergo prospective validation.

Keywords: Orchialgia; chronic scrotal content pain; testicular diseases; testicular pain; chronic pain

Submitted Feb 11, 2018. Accepted for publication Apr 03, 2018.

doi: $10.21037 /$ tau.2018.04.10

View this article at: http://dx.doi.org/10.21037/tau.2018.04.10 


\section{Introduction}

Chronic scrotal content pain, or orchialgia, is a significant source of morbidity for patients, and a common complaint in those presenting to the urologist. Many urologists see such patients on a near weekly basis (1). Impact on quality of life (QOL) can be significant with one study showing median symptom duration of 4.9 years with $30 \%$ of patients claiming to make significant adjustments to their life because of the disease (2). Despite this, high quality research into etiology, impact and therapy has been scant relative to other urologic conditions.

One limitation to clinical research in orchialgia is the absence of a validated way to measure symptom severity. The development of validated symptom scores is especially important in subjective disorders; development of such instruments in voiding dysfunction, sexual dysfunction and prostatitis has led to more widespread scientific study in these conditions. Most studies to date have either used a trinary "gone", "improved" or "unchanged" outcome (3) or a pain scale not validated for orchialgia (4). This may fail to adequately encompass the patient experience in chronic pain disorders, which can represent a complex interaction of a constellation of symptoms. We wished therefore to develop a clinically relevant symptom index to improve the assessment and study for patients with chronic scrotal content pain/orchialgia.

\section{Methods}

After IRB approval, we developed a comprehensive initial questionnaire that included 70 questions encompassing medical history, pain and associated symptoms. Because the surveys were de-identified, no signed informed consent was required. These were selected based on interviews with specialists in the field and patients. History questions included symptom duration, prior testicular surgeries (including vasectomy), testicular trauma, infections, peripheral neuropathy and undescended testicle. Pain questions included minimum and maximum levels, location including multiple sites outside the testicle or groin, nature (burning, numbness) and associated swelling. Urinary questions included frequency, urgency, hesitancy and dysuria. Sexual questions included erection quality, premature ejaculation, painful ejaculation and decreased libido. QOL questions included impact on work, recreation, how often they thought about the pain and how they would feel if their current condition was unchanged.
Continuous variables were ranked from $0-10$ in severity and a bothersome scale was associated with each symptom question as a weight for further item selection.

These questionnaires were then distributed at two high volume orchialgia centers, Cleveland Clinic in Cleveland, $\mathrm{OH}$ and The Personalized Urology and Robotics (PUR) Clinic in Winterhaven, FL. To be included in the study, patients needed to have primarily scrotal content pain, and could not have any obvious reversible pathology such as acute infection, hydrocele, torsion, testis cancer, etc. The questionnaires were then collected and data was pooled. We did not collect data on patients who refused to complete the questionnaire.

Results were presented as means with standard deviations and medians with interquartile range (IQR) for continuous variables or variables scaled with multiple levels. For binary variables, percentages and proportions were given. All analyses and graphics were done using the statistical software package $\mathrm{R}$ version 3.2.1 (R Development Core Team, www.r-project.org).

We applied psychometric principles for item selection to generate the final questionnaire. The agglomerative hierarchical clustering function in the package cluster was used for a "bottom up" visualization of individual items forming clusters based on the item-observation dissimilarity matrix. Items clinically less relevant to orchialgia in a shared cluster with the same underlying construct were removed, for example, when pain in the abdomen and pain in the groin were both in the same cluster the former was excluded. The bother scale for a corresponding item intended as a weight of the item was removed with the item or without the item depending on the significance of the item.

Principal component analysis (PCA) and the Scree test (5) in the R Stats Package were used for the selection of the number of factors, i.e., groups of items with the same underlying construct, to be included in the final questionnaire. The Scree test ranks factors based on the variation in the data a factor explains. The R Psych Package developed by Revelle (http://personality-project. org/r/psych-manual.pdf; https://CRAN.R-project.org/ package $=$ psych, Version 1.7.8) was used for the final instrument construction and evaluation of its internal consistency and construct validity. The ICLUST procedure in the Psych Package was specifically developed for clustering questionnaire items, while the principal procedure was used to identify subdomains by examining factor loadings termed eigenvalues which, when the correlation matrix was not rotated, equals to the variation in 


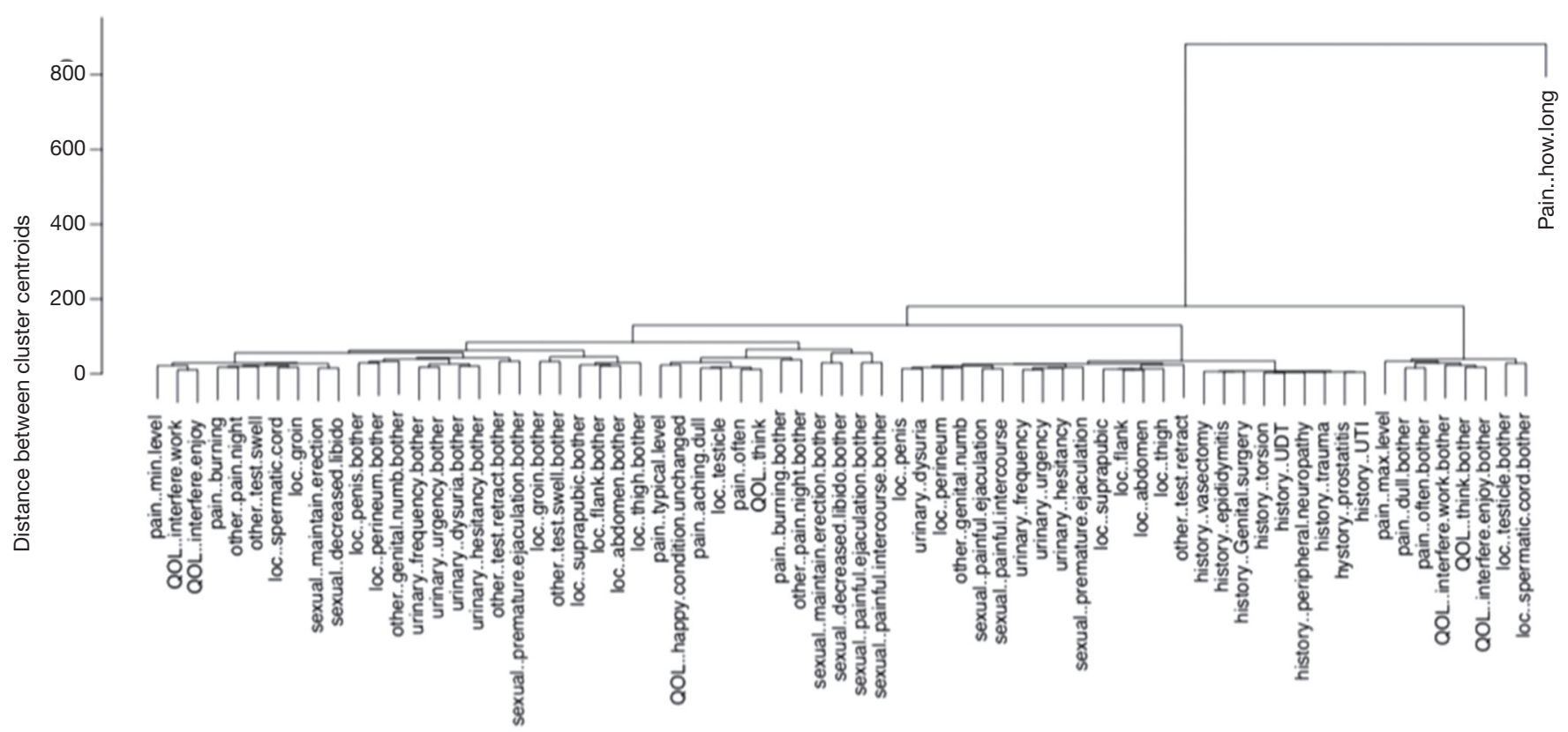

Figure 1 Dendrogram illustrating agglomerative hierarchical cluster analysis used to optimize 70-item pilot questionnaire based on patient responses.

the data attributed to the factor. For the final questionnaire, the number of factors was based on the variation explained by the last factor of at least $20 \%$, the standard eigenvalue of an item close to 0.5 or above and the chi-square test for component sufficiency.

\section{Results}

A total of 113 patients completed the 70 -item questionnaire. No questions were answered by fewer than $60 \%$ of the participants. Median symptom duration was 12 months (range, 3-336 months). Outside the testicle, pain was felt in the spermatic cord $(66 \%)$, groin $(66 \%)$, penis $(24 \%)$, suprapubic region (38\%), flank (31\%), thigh (32\%), abdomen (36\%) and perineum (35\%). Bother scores were high only for testicle and spermatic cord pain. Description of the pain as burning and being awoken at night was less common, but when present had high bother scores. Urinary frequency was common (54\%) but not bothersome. Sexual dysfunction was surprisingly common: $55 \%$ had erectile dysfunction, $56 \%$ had decreased libido and 39\% had painful ejaculation with high bother for all.

Multiple analyses resulted in the final 12-item instrument with variable reduction from an initial 70-item pilot questionnaire. Clustering was used for exploratory analyses to examine relatedness of the items on the questionnaire
(Figure 1). Items with predominate influence on the hierarchical clustering, such as duration of symptoms and maximum level of pain, were excluded first. The bother scale intended as a weight for each corresponding item was eventually removed along with insignificant items or excluded when an item was selected. Cluster analysis suggested a 3 -factor final instrument and the Scree test indicated a dominate first two factors followed by a third factor with the eigenvalue slightly $>1.0$. PCA showed that both the 3 -factor and the 2 -factor instruments were sufficient (chi-square test, $\mathrm{P}<0.001$ ). All 12 items had factor loadings near or greater than 0.5 on the basis of the 2 -factor model (Table 1). The final candidate questionnaire consisted of three groups of chronic orchialgia symptoms: pain, sexual dysfunction, and QOL (Figure 2). The three QOL variables (pain impacts on work, interferes with leisure activities, persists for life) were tightly clustered together regardless of whether the instrument was of 3- or 2-factor. The QOL parameters clustered with minimum level, typical level, and pain at night in one analysis, and with ED, low libido and painful ejaculation in another analysis.

\section{Discussion}

Chronic orchialgia/scrotal contents pain is common, poorly understood and under-studied (6). A significant barrier to 
Table 1 The Chronic Orchialgia Symptom Index (COSI): item listings, PCA factor loadings, and scoring statistics

\begin{tabular}{|c|c|c|c|}
\hline Item & Scoring & Factor loadings & Median (IQR) \\
\hline Pain in groin (0 no, 1 yes) & $0-1$ & 0.64 & $1(0,1)$ \\
\hline Pain that wakes up in the night ( 0 none, 1 some, 2 always) & $0-2$ & 0.55 & $1(0,1.5)$ \\
\hline Minimum pain in the past week ( 0 none -5 worst pain imaginable) & $0-5$ & 0.59 & $1(0,2)$ \\
\hline How often ( 0 none, 1 occasional, 2 usually, 3 always) & $0-3$ & 0.64 & $2(2,3)$ \\
\hline Subtotal pain score & $0-17$ & & $8(5,10)$ \\
\hline Erectile dysfunction [0 none, 1 some, 2 always (cannot maintain erection)] & $0-2$ & 0.60 & $1(0,1)$ \\
\hline Low libido (0 no, 1 yes) & $0-1$ & 0.49 & $1(0,1)$ \\
\hline QOL interfere with work (0 not at all-5 always) & $0-5$ & 0.76 & $2(0,3)$ \\
\hline QOL interfere with leisure ( 0 not at all- 5 always) & $0-5$ & 0.74 & $3(1,4)$ \\
\hline QOL same way rest of your life ( 0 delight -5 terrible) & $0-5$ & 0.76 & $5(4,5)$ \\
\hline Subtotal QOL score & $0-15$ & & $9(4,12)$ \\
\hline Total COSI score & $0-37$ & & $19(12,24.5)$ \\
\hline
\end{tabular}

the scientific evaluation of a condition and its treatment is the lack of a standardized and validated way to assess and quantify symptoms. Currently, the orchialgia literature uses subjective measures of patient improvement, visual analogue scales for pain or other pain instruments not designed or validated for this type of pain such as the PIQ-6 (4). Furthermore, simple pain scales may fail to incorporate other features of the condition that also impact QOL. For example, the NIH Chronic Prostatitis Symptom Index (CPSI) (7) incorporates questions on pain, urinary bother and QOL. In 2002 Nickel et al. developed an Epididymitis Symptom Index, which was based on the CPSI, but was never validated and was subsequently not widely used (2).

There are a wide variety of proposed treatments for chronic orchialgia, including anti-inflammatories, physical therapy, gabapentin, epididymectomy, microsurgical denervation, orchiectomy, and botulinum toxin injection $(3,8,9)$. For patients with orchialgia following vasectomy, there are several different surgical treatments: vasectomy reversal, granuloma excision, microsurgical denervation and epididymectomy (9-11). The lack of a validated questionnaire makes direct comparison of these techniques difficult, and is perhaps partially to blame for lack of evidence-based guidelines.

There were a number of interesting observations in our patient cohort. For a condition that affects younger men, the incidence of sexual symptoms including ED was high and had high bother. This has been seen in prior studies of orchialgia (12) and pain in testis cancer survivors (13). Urinary symptoms were common, which has not been previously reported, but the bother was low. During the PCA, a separate distinct cluster of men with urinary symptoms, penile and perineal pain, thigh pain, pain with ejaculation and genital numbness was identified. This suggests a subset of patients with pelvic floor spasm (14) who may be best treated with physical therapy (15).

Limitations to the study include modest sample size given the number of variables, using patients only seen at specialty centers and lack of a gold standard comparator. The next step is to validate this candidate instrument. It will be critical to determine whether it is representative of a variety of orchialgia patients, is reproducible and is responsive to therapy. These studies are ongoing. 
Chronic Orchialgia Symptom Index

Pain Symptoms

1. When you have testicle pain, do you also feel it in your groin (area above testicle)?

No__(0) Yes__(1)

2. Would you describe your testicle pain as burning?

No _ (0) Yes _ (1)

3. Does your testicular pain wake you up at night?

Never __(0), Sometimes _(1) Always __ (2)

4. In the past week how often did you feel pain in your testicle?

Never__(0), Occasionally _(1), Usually _(2), Always _(3)

5. What number best describes your MINIMUM (lowest) testicle pain in the past week?

0 _ $\quad 1_{-} \quad 2_{-} \quad 3_{-} \quad 4_{-} \quad 5_{-}$

None Mild Worst pain imaginable

6. What number best describes your MAXIMUM (highest) testicle pain in the past week?

$0 \ldots \quad 1_{-} \quad 2_{-} \quad 3_{-} \quad 4_{-} \quad$ 5_

None Mild $\quad-$ Worst pain imaginable

\section{Sexual Symptoms}

7. In the past week how often have you had difficulty achieving or maintaining an erection?

Never (eg. you have normal erections) _ (0), Sometimes _(1), Always _(2)

8. In the past week, has your desire to have sex (libido) been lower than normal for you?

No _ (0), Yes_(1)

9. In the past week have sexual activities been painful?

No _ (0), Sometimes __(1), Always __(2)

\section{Quality of Life}

10. In the past week, how much has your testicular pain prevented you from working or doing your normal daytime activities?

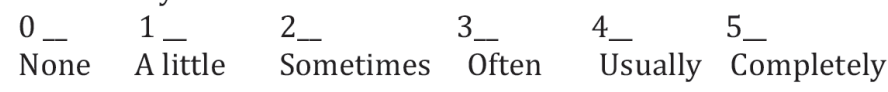

11. In the past week, how much has your testicular pain prevented you from doing leisure activities you enjoy?

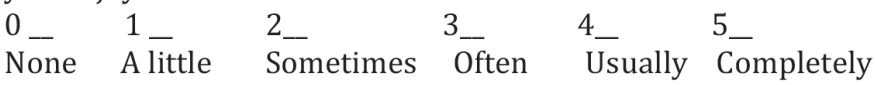

12. If nothing changed and your symptoms remained this way for the rest of your life, how would you feel?

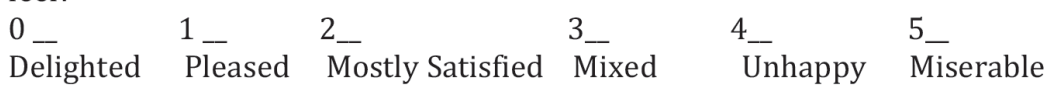

Score: Pain (Q 1-6) _ Sexual (Q 7-9) __ QOL (Q 10-12) _

Figure 2 Final Chronic Orchialgia Symptom Index (COSI) derived from initial 70-item questionnaire. 


\section{Acknowledgements}

None.

\section{Footnote}

Conflicts of Interest: The authors have no conflicts of interest to declare.

Ethical Statement: The primary IRB was Cleveland Clinic which approved the multicenter study. There was an individual data share agreement between institutions for sharing the de-identified data IRB 16-195, Cleveland Clinic IRB. Because the surveys were de-identified, no signed informed consent was required.

\section{References}

1. Strebel RT, Leippold T, Luginbuehl T, et al. Chronic scrotal pain syndrome: management among urologists in Switzerland. Eur Urol 2005;47:812-6.

2. Nickel JC, Siemens DR, Nickel KR, et al. The patient with chronic epididymitis: characterization of an enigmatic syndrome. J Urol 2002;167:1701-4.

3. Levine LA, Matkov TG. Microsurgical denervation of the spermatic cord as primary surgical treatment of chronic orchialgia. J Urol 2001;165:1927-9.

4. Calixte N, Tojuola B, Kartal I, et al. Targeted Robotic Assisted Microsurgical Denervation of the Spermatic Cord for the Treatment of Chronic Orchialgia or Groin Pain: A Single Center, Large Series Review. J Urol 2018;199:1015-22.

5. Cattell RB. The Scree Test For The Number Of Factors. Multivariate Behav Res 1966;1:245-76.

Cite this article as: Polackwich AS, Arora HC, Li J, Levine L, Tojuola B, Parekattil S, Shoskes DA. Development of a clinically relevant symptom index to assess patients with chronic orchialgia/chronic scrotal content pain. Transl Androl Urol 2018;7(Suppl 2):S163-S168. doi: 10.21037/tau.2018.04.10
6. Sigalos JT, Pastuszak AW. Chronic orchialgia: epidemiology, diagnosis and evaluation. Transl Androl Urol 2017;6:S37-S43.

7. Litwin MS, McNaughton-Collins M, Fowler FJ Jr, et al. The National Institutes of Health chronic prostatitis symptom index: development and validation of a new outcome measure. Chronic Prostatitis Collaborative Research Network. J Urol 1999;162:369-75.

8. Calixte N, Brahmbhatt J, Parekattil S. Chronic Testicular and Groin Pain: Pathway to Relief. Curr Urol Rep 2017;18:83.

9. Lee JY, Lee TY, Park HY, et al. Efficacy of epididymectomy in treatment of chronic epididymal pain: a comparison of patients with and without a history of vasectomy. Urology 2011;77:177-82.

10. Polackwich AS, Tadros NN, Ostrowski KA, et al. Vasectomy Reversal for Postvasectomy Pain Syndrome: A Study and Literature Review. Urology 2015;86:269-72.

11. Tan WP, Levine LA. Micro-Denervation of the Spermatic Cord for Post-Vasectomy Pain Management. Sex Med Rev 2018;6:328-34.

12. Ciftci H, Savas M, Gulum M, et al. Evaluation of sexual function in men with orchialgia. Arch Sex Behav 2011;40:631-4.

13. Pühse G, Wachsmuth JU, Kemper S, Husstedt IW, et al. Chronic pain has a negative impact on sexuality in testis cancer survivors. J Androl 2012;33:886-93.

14. Polackwich AS, Li J, Shoskes DA. Patients with Pelvic Floor Muscle Spasm Have a Superior Response to Pelvic Floor Physical Therapy at Specialized Centers. J Urol 2015;194:1002-6.

15. Farrell MR, Dugan SA, Levine LA. Physical therapy for chronic scrotal content pain with associated pelvic floor pain on digital rectal exam. Can J Urol 2016;23:8546-50. 